\title{
Moderate sleep apnoea: a "silent" disorder, or not a disorder at all?
}

\author{
Edward O. Bixler ${ }^{1}$, Alexandros N. Vgontzas ${ }^{1}$, Jordan Gaines ${ }^{1}$, \\ Julio Fernandez-Mendoza ${ }^{1}$, Susan L. Calhoun ${ }^{1}$ and Duanping Liao ${ }^{2}$
}

Affiliations: 'Sleep Research and Treatment Center, Dept of Psychiatry, Penn State University College of Medicine, Hershey, PA, USA. ${ }^{2}$ Dept of Public Health Sciences, Penn State University College of Medicine, Hershey, PA, USA.

Correspondence: Edward O. Bixler, Penn State College of Medicine, Psychiatry, 500 University Drive, Hershey, PA 17033, USA. E-mail: ebixlerQhmc.psu.edu

0 @ERSpublications

New ERJ study adds to the evidence casting doubt on the utility of recommended cut-offs for sleep apnoea treatment http://ow.ly/VgjBL

Most sleep clinicians and scientists will agree that if a patient with sleep apnoea is symptomatic and has an apnoea/hypopnea index (AHI) of more than 15 events per hour, he or she deserves treatment. However, there is a large group of patients with mild and moderate sleep apnoea who are asymptomatic. For these individuals, the decision of when to treat is a grey area, and current guidelines leave clinicians wondering what to do.

ARNARDOTtir et al. [1] from the University Hospital of Iceland studied a large general population sample of middle-aged individuals using polysomnographic sleep recordings, questionnaires and the psychomotor vigilance test (PVT). Of the 415 subjects, $12.5 \%$ had moderate obstructive sleep apnoea (OSA; AHI between 15 and 30$), 2.9 \%$ had severe OSA $(\mathrm{AHI} \geqslant 30)$, and $3.6 \%$ were already diagnosed and treated for OSA. Interestingly, no relationship was found between AHI and subjective sleepiness or clinical symptoms, whereas a relationship with objective vigilance, as measured by the PVT, was found only with those with $\mathrm{AHI} \geqslant 30$. Furthermore, subjects with moderate or severe OSA were not more likely to have cardiometabolic disease than subjects with no or mild OSA. These results raise the question of whether the current cut-off of $\mathrm{AHI} \geqslant 15$ as a sole criterion is appropriate for diagnosis and treatment of OSA.

In 1998, we first reported, using the Penn State Adult Cohort, a different frequency and pattern of prevalence of OSA based on AHI solely versus based on a combination of AHI and presence of clinical symptoms (i.e. sleepiness and/or cardiometabolic disorders) [2]. The prevalence of OSA based on AHI alone was two to three times the prevalence of OSA based on laboratory and clinical criteria. Furthermore, based on AHI alone, the prevalence of OSA increased linearly, reaching its peak at very old age, whereas the prevalence based on combined criteria peaked at the age of 55 years for men and 65 years for women, then declined steeply (figure 1) [2,3]. In addition, oxygen desaturation in older individuals was significantly less compared to that in younger individuals, suggesting the possibility that OSA in the elderly is less severe. Together, these findings suggested that the sleep field should re-evaluate the current criteria for diagnosis and treatment in this age group.

These early observations were further strengthened by another report in the same large cohort on the association of hypertension and OSA. We reported that AHI, controlled for relevant confounding variables, was independently associated with hypertension [4]. In addition, this study confirmed that the strength of the association between AHI and blood pressure was strongest in the youngest (figure 2). These findings further supported the implication that OSA in older individuals is not an independent risk factor for hypertension.

Received: Nov 232015 | Accepted: Nov 272015

Conflict of interest: None declared.

Copyright @ERS 2016 


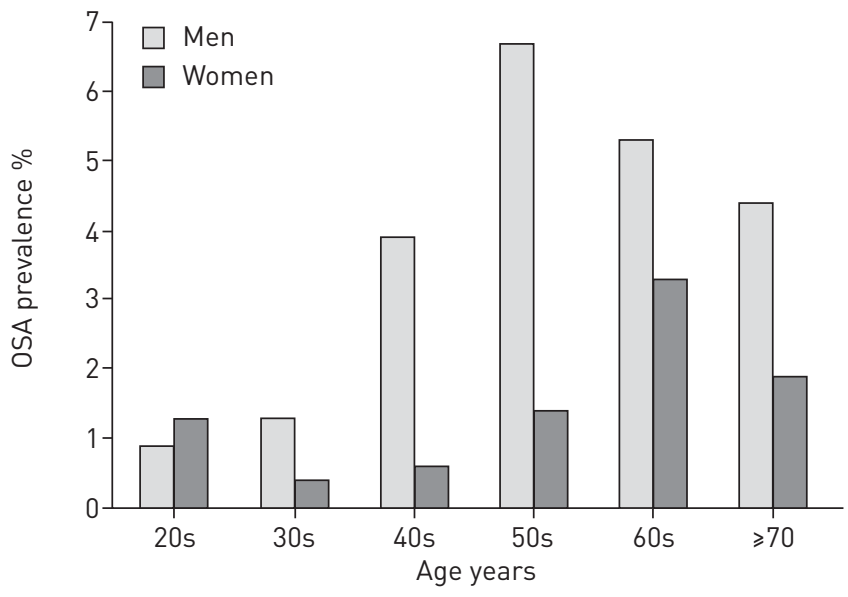

FIGURE 1 Age distribution of prevalence of obstructive sleep apnoea (OSA) by decade lapnoea/ hypopnoea index $\geqslant 15$ and presence of daytime symptoms). Prevalence peaks at age 55 years for men and 65 years for women. Data adapted from BIXLER et al. [2] and BIXLER et al. [3].

The findings from prospective studies are equally interesting and surprising, particularly in terms of mortality risk. In an 18-year follow-up of the Wisconsin Sleep Cohort [5], an increased hazard ratio was reported only for severe OSA $(\mathrm{AHI} \geqslant 30)$, whereas the hazard ratio for mortality associated with moderate OSA (AHI $\geqslant 15$ but $<30$ ), was negligible. Similar findings were reported in an 8-year follow-up of the Sleep Heart Health Study [6]. Furthermore, in an elderly cohort ( $\geqslant 65$ years), LAVIE and LAVIE [7] reported that mortality was not increased, even in those with respiratory disturbance index (RDI) $>40$ events per hour, whereas moderate OSA (RDI $\geqslant 20$ but $<40$ ) was even associated with an "unexpected survival advantage." In another clinical cohort of men with OSA that were followed for 10 years, no association was found between mild/moderate OSA $(\mathrm{AHI} \geqslant 5$ but $<30)$ and mortality [8]. Combined, these results suggest that untreated moderate OSA is not associated with mortality, even after a long follow-up period (up to 20 years); in fact, in the elderly, moderate OSA might even have a protective effect [7].

The findings of prospective studies on the association of moderate OSA with cardiometabolic disorders are inconsistent and of rather modest impact. In the Sleep Heart Health Study, OSA was significantly associated with incident stroke only in men whose AHI was $>20$, but not in women in that apnoea range [9]. In the Wisconsin Sleep Cohort, the incidence of stroke was not associated with an AHI of $<20$, whereas it was significantly elevated in those subjects with $\mathrm{AHI} \geqslant 20[10]$. In terms of cardiovascular problems, the Sleep Heart Health Study reported that incident coronary heart disease and heart failure were not significantly associated with moderate OSA (AHI $\geqslant 15$ but $<30$ ), though there was a significant association with AHI $\geqslant 30$ in men $\leqslant 70$ years old; however, the risk of these cardiac problems was not increased for older men or women, even with an $\mathrm{AHI} \geqslant 30$ [11]. In the Wisconsin Sleep Cohort, an $\mathrm{AHI} \geqslant 15$ was not associated with a significant risk for diabetes 4 years after the baseline measure [12]. In contrast with these findings, mild-to-moderate OSA was associated with a significant risk for development of hypertension in this cohort [13]; however, the potential confounding effects of age, gender and obesity were not examined. Also from the Wisconsin Sleep Cohort, minimum (but not mean) arterial oxygen saturation measured by pulse oximetry $\left(\mathrm{SPO}_{2}\right)$ was associated with future carotid plaques. Interestingly, in this study, the use of

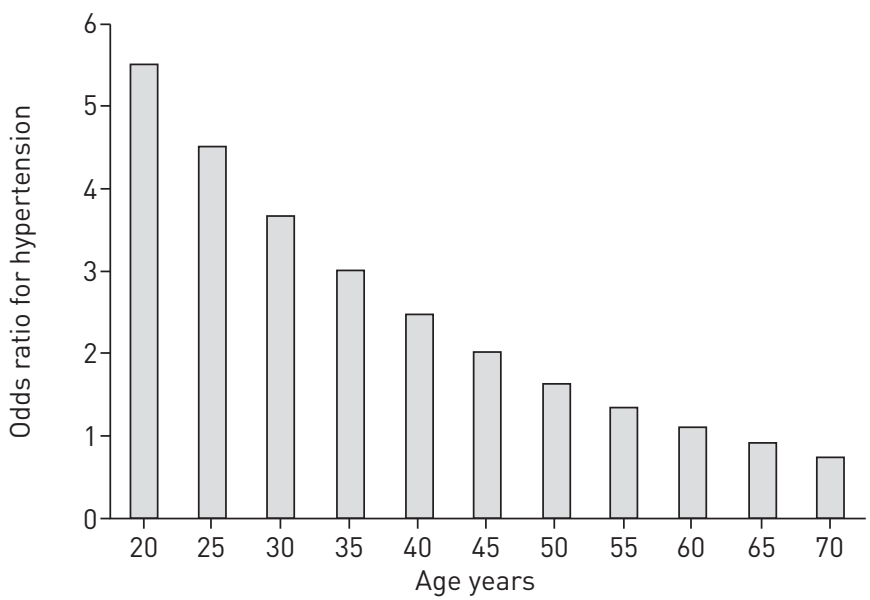

FIGURE 2 Age distribution of the odds of hypertension by decade with the presence of apnoea/hypopnoea index $\geqslant 15$, adjusting for age, body mass index, gender, menopause and/or hormone replacement therapy. Data adapted from BIXLER et al. [4]. 
continuous positive airway pressure (CPAP) did not change the association between minimum $\mathrm{SpO}_{2}$ and carotid atherosclerosis [14]. We should note that treatment of OSA with CPAP does not improve inflammation in these patients [15-17], which suggests that the association between OSA and hypertension or carotid atherosclerosis may be mediated by inflammation itself and not apnoeas/hypopneas. There is currently a gap in the literature, with a lack of studies on the association of moderate OSA in the presence of comorbid cardiometabolic disorders, as well as future fatal and non-fatal health sequelae.

Another concern is the association of sleepiness and impaired performance with OSA. In the present study by ARNARDOTTIR et al. [1], no association was found between sleepiness and OSA, whereas an effect on performance was found only in those with an $\mathrm{AHI} \geqslant 30$. This is consistent with other population samples that have reported that only one out of five people with OSA complain of sleepiness [18-20]. Nevertheless, sleepiness is a more frequent symptom in clinical samples and of concern to patients, clinicians and public health authorities. A factor to consider in understanding this association is the evidence that other comorbid factors, such as obesity, are much stronger risk factors than AHI for excessive daytime sleepiness $[19,21]$. In a large population cohort, it has been shown that obesity, depression, diabetes and young age are much stronger risk factors for daytime sleepiness than AHI [19]. Furthermore, obesity and depression, but not OSA, are risk factors for incident daytime sleepiness [22]. This may explain why, in clinical populations of apnoeics, the majority of the patients are obese, young to middle age, and depressed in contrast to the general population cohorts.

Obesity has been well established as a contributing factor for clinical outcomes associated with AHI. However, there is also strong evidence that metabolic aberrations related, but not exclusive, to obesity (i.e. inflammation, insulin resistance) are the primary mechanistic pathway. An early finding demonstrated in several population samples that pre-menopausal women were protected from OSA, and that post-menopausal women on hormone replacement therapy continued to be protected $[3,23,24]$. In a secondary analysis of a study in the Women's Health Initiative, it has been shown that women randomised to hormone replacement therapy had reduced insulin resistance [25]. In addition, several studies have reported a high risk for both OSA and excessive daytime sleepiness in women with polycystic ovary syndrome, a condition associated with hyperandrogenism and insulin resistance [26, 27]. These findings support the position that metabolic factors associated with gender play a more significant role than AHI in the association of OSA with clinical outcomes.

It is now widely accepted that the metabolic syndrome is the mechanistic link between AHI and cardiovascular outcomes, suggesting that central obesity (e.g. waist circumference) may be a better surrogate marker of cardiovascular risk than body mass index. Visceral adiposity, inflammation and insulin resistance, key components of the underlying pathophysiology of the metabolic syndrome, are the pathways leading to OSA or linking OSA to its cardiometabolic sequelae. It has also been proposed that OSA is one of the manifestations of the so-called metabolic syndrome [28]. Inflammation and metabolic aberrations are independently associated with many of the outcomes examined in OSA (i.e. sleepiness, fatigue and cardiometabolic disorders). We should note that preclinical markers of cardiometabolic disease, such as visceral adiposity, inflammation and insulin resistance, have not been measured or taken into account in any cross-sectional or prospective study to control for the net effect of the apnoeas/hypopneas.

In conclusion, the study by ARNardotTir et al. [1] adds to the evidence that cast doubt on the utility of the currently recommended cut-offs for treatment of OSA $(\mathrm{AHI} \geqslant 15)$, even in the absence of any clinical problems (ICSD-3, DSM-V). Certainly, these cut-offs are inappropriate for older men and women as well as asymptomatic patients. In fact, the widely used, cumbersome mechanical treatments, such as positive airway pressure, may not be useful in this population. Longitudinal studies assessing the risk of OSA in the presence of comorbid conditions and the use of preclinical biological markers, such as inflammation markers, may help us to define who of the individuals with moderate, "silent" OSA need protective interventions. Finally, from a therapeutic standpoint, the elimination of AHI should not be our only concern. Rather, addressing modifiable risk factors associated with OSA and cardiometabolic health, such as obesity, exercise and mood, may narrow the grey area for practising clinicians.

\section{References}

1 Arnardottir ES, Bjornsdottir E, Olafsdottir KA, et al. Obstructive sleep apnoea in the general population: highly prevalent but minimal symptoms. Eur Respir J 2016; 47: 194-202.

2 Bixler EO, Vgontzas AN, Ten Have T, et al. Effects of age on sleep apnea in men. I. Prevalence and severity. Am J Respir Crit Care Med 1998; 157: 144-148.

3 Bixler EO, Vgontzas AN, Lin HM, et al. Prevalence of sleep-disordered breathing in women: effects of gender. Am J Respir Crit Care Med 2001; 163: 608-613.

4 Bixler EO, Vgontzas AN, Lin H-M, et al. Association of hypertension and sleep disordered breathing. Arch Intern Med 2000; 160: 2289-2295.

5 Young T, Finn L, Peppard PE, et al. Sleep disordered breathing and mortality: eighteen-year follow-up of the Wisconsin Sleep Cohort. Sleep 2008; 31: 1071-1078. 
6 Punjabi NM, Caffo BS, Goodwin JL, et al. Sleep-disordered breathing and mortality: a prospective cohort study. PLOS 2009; 6: e1000132.

7 Lavie P, Lavie L. Unexpected survival advantage in elderly people with moderate sleep apnoea. J Sleep Res 2009; 18: 397-403.

8 Marin JM, Carrizo S, Vicente E, et al. Long-term cardiovascular outcomes in men with obstructive sleep apnoea-hypopnoea with or without treatment with continuous positive airway pressure: an observational study. Lancet 2005; 365: 1045-1053.

9 Redline S, Yenokyan G, Gottlieb DJ, et al. Obstructive sleep apnea-hypopnea and incident stroke: the Sleep Heart Health Study. Am J Respir Crit Care Med 2010; 182: 269-277.

10 Arzt M, Young T, Finn L, et al. Association of sleep-disordered breathing and the occurrence of stroke. Am J Respir Crit Care Med 2005; 172: 1447-1451.

11 Gottlieb DJ, Yenokyan G, Newman AB, et al. Prospective study of obstructive sleep apnea and incident coronary heart disease and heart failure: the Sleep Heart Health Study. Circulation 2010; 122: 352-360.

12 Reichmuth KJ, Austin D, Skatrud JB, et al. Association of sleep apnea and type II diabetes: a population-based study. Am J Respir Crit Care Med 2005; 172: 1590-1595.

13 Peppard PE, Young T, Palta M, et al. Prospective study of the association between sleep-disordered breathing and hypertension. N Engl J Med 2000; 342: 1378-1384.

14 Gunnarsson SI, Peppard PE, Korcarz CE, et al. Minimal nocturnal oxygen saturation predicts future subclinical carotid atherosclerosis: the Wisconsin Sleep Cohort. J Sleep Res 2015; 24: 680-686.

15 Jullian-Desayes I, Joyeux-Faure M, Tamisier R, et al. Impact of obstructive sleep apnea treatment by continuous positive airway pressure on cardiometabolic biomarkers: a systematic review from sham CPAP randomized controlled trials. Sleep Med Rev 2015; 21: 23-38.

16 Vgontzas A, Zoumakis E, Bixler EO, et al. Selective effects of CPAP on sleep apnoea-associated manifestations. Eur I Clin Invest 2008; 38: 585-595.

17 Kritikou I, Basta M, Vgontzas AN, et al. Sleep apnea, sleepiness, inflammation, and insulin resistance in middle-aged males and females. Eur Respir J 2014; 43: 145-155.

18 Young T, Palta M, Dempsey J, et al. The occurrence of sleep-disordered breathing among middle-aged adults. N Engl J Med 1993; 328: 1230-1235.

19 Bixler EO, Vgontzas AN, Lin HM, et al. Excessive daytime sleepiness in a general population sample: the role of sleep apnea, age, obesity, diabetes, and depression. J Clin Endocrinol Metab 2005; 90: 4510-4515.

20 Kapur VK, Baldwin CM, Resnick HE, et al. Sleepiness in patients with moderate to devere sleep-disordered breathing. Sleep 2005; 28: 472-477.

21 Vgontzas AN, Bixler EO, Tan TL, et al. Obesity without sleep apnea is associated with daytime sleepiness. Arch Intern Med 1998; 158: 1333-1337.

22 Fernandez-Mendoza J, Vgontzas AN, Kritikou I, et al. Natural history of excessive daytime sleepiness: role of obesity, weight loss, depression and sleep propensity. Sleep 2015; 38: 351-360.

23 Young T, Rabago D, Zgierska A, et al. Objective and subjective sleep quality in premenopausal, perimenopausal, and postmenopausal women in the Wisconsin Sleep Cohort Study. Sleep 2003; 26: 667-672.

24 Shahar E, Redline S, Young T, et al. Hormone replacement therapy and sleep-disordered breathing. Am J Respir Crit Care Med 2003; 167: 1186-1192.

25 Margolis KL, Bonds DE, Rodabough RJ, et al. Effect of oestrogen plus progestin on the incidence of diabetes in postmenopausal women: results from the Women's Health Initiative Hormone Trial. Diabetologia 2004; 47: $1175-1187$.

26 Vgontzas AN, Legro RS, Bixler EO, et al. Polycystic ovary syndrome is associated with obstructive sleep apnea and daytime sleepiness: role of insulin resistance. J Clin Endocrinol Metab 2001; 86: 517-520.

27 Tasali E, Van Cauter E, Ehrmann DA. Relationships between sleep disordered breathing and glucose metabolism in polycystic ovary syndrome. J Clin Endocrinol Metab 2006; 91: 36-42.

28 Vgontzas AN, Bixler EO, Chrousos GP. Sleep apnea is a manifestation of the metabolic syndrome. Sleep Med Rev 2005; 9: 211-214. 